\title{
Imaging- guided facet joint injection technique for lower back pain management
}

\author{
Abdelghany Mohammed Motawea, Mohammed Ramadan El Kholy, Osama Mohamed Ebied, \\ Waleed Abd El Fattah Mousa, Mohamed Shawky Abdullah
}

Radiodiagnosis Department, Faculty of Medicine, Menoufia University, Shebin El Kom, Egypt

Email address:

abdelghany8000@gmail.com (A. M. Motawea),dr_melkholy@hotmail.com (M. R. E. Kholy), Osamaebied@hotmail.com (O. M. Ebied), Waleedmousa75@gmail.com (W. A. Mousa),M.shawky76@yahoo.com (M. S. Abdullah)

\section{To cite this article:}

Abdelghany Mohammed Motawea, Mohammed Ramadan El Kholy, Osama Mohamed Ebied, Waleed Abd El Fattah Mousa, Mohamed Shawky Abdullah. Imaging- Guided Facet Joint Injection Technique for Lower Back Pain Management. American Journal of Health Research. Vol. 3, No. 2, 2015, pp. 52-56. doi: 10.11648/j.ajhr.20150302.11

\begin{abstract}
Objectives: This study aimed to evaluate the role of image-guided lumbar facet joint injection in lower back pain. Background: With the increased lifetime prevalence of spinal pain and the realization by surgeons that not all backache is a 'disc' and not all patients with LBP will respond to surgery, there is increasing awareness of the role of percutaneous injection techniques in the nonoperative management of chronic LBP. The use of image-guidance with fluoroscopy or CT scan has increased the precision and safety of these procedures. Methods: Fifty patients with chronic LBP of more than 3 months' duration were selected for facet joint infiltration. Facet joint injections were carried out under fluoroscopic guidance in 38 patients and under CT guidance in 12 cases. Pain relief was assessed using the visual analog scale at 4, 12 , and 24 weeks postprocedure. These results were compared to a control group $(\mathrm{n}=25)$ which were subjected to medical treatment. Results: A total of 146 facet joints were infiltrated in 50 patients over a 2-year period. There was significant pain relief in $88 \%$ after 4 weeks, in $92 \%$ after 12 weeks, and in $64 \%$ after 24 weeks. No major complications were encountered. The control group which was subjected to medical treatment showed pain relief in $40 \%$ after 4 weeks, in $32 \%$ after 12 weeks, and in $28 \%$ after 24 weeks with less pain relief in comparison to the injected group. Conclusion: With intraarticular facet joint injections, the evidence for short- and medium-term pain relief is successful for lumbar pain in comparison to medical treatment. Facet nerve block was found to be a simple, minimally invasive, and safe procedure. With precise patient selection, we achieved long-term success rates of over $60 \%$ after 24 weeks. We conclude that this method represents an important alternative treatment for nonradicular back pain.
\end{abstract}

Keywords: Facetal Arthropathy, Facetal Infiltrations, Facetal Block, Low Back Pain

\section{Introduction}

Chronic low back pain (LBP) has assumed endemic proportions, with an annual prevalence of $5-20 \%$ in the industrialized world ${ }^{(1)}$. It is not always possible to pinpoint the exact structure or pathology responsible for LBP. In 2001, a large study on patients with chronic LBP showed that in as many as $15-45 \%$ of patients the pain was due to pathology of the facet joints and only in $13-20 \%$ was the pain due to herniated discs ${ }^{(2,3)}$. With the realization by surgeons that not all backache is a 'disc' and not all patients with LBP will respond to surgery, there is increasing awareness of the role of percutaneous injection techniques in the nonoperative management of chronic LBP. The use of image-guidance with fluoroscopy or CT scan has increased the precision and safety of these procedures ${ }^{(4)}$.

\section{Material and Methods}

In addition to the control group ( 25 patients subjected to medical treatment), a total of 50 patients were included in the study on the basis of certain criteria like chronic LBP of more than 3 months' duration with a clinically suspected facet joint component not evident by $\mathrm{CT}$, preoperative assessment for patients undergoing disc surgery who have facet arthropathy evident by $\mathrm{CT}$ or pseudoradicular manifestation to exclude a facet source of pain, chronic post operative pseudoradicular pain, failed back surgery 
syndrome (laminectomy syndrome), facet joint defects, synovial cysts, unilateral facet arthropathy, one level facet joint arthropathy. All patients were initially assessed by a neurosurgeon before referral for the facet joint block

The exclusion criteria were lumbar spinal deformities, severe foraminal stenosis, evidence of nerve root compression at the expected level on MRI, Clinical or imaging evidence of infection or neoplastic disease, Possible pregnancy, bleeding diathesis, or anticoagulant therapy, History of sensitivity to local anesthetics

There were 26 men and 24 women included in the study; the ages ranged from $34-55$ years. The duration of symptoms varied from 3 months to 2.5 years (mean duration: 11 months). The procedure was explained to the patient in detail and written consent was obtained. Injections were performed under fluoroscopic guidance in most (38/50) patients. CT guidance was used in twelve cases. The levels and side(s) to be injected were selected by the treating pain physician on the basis of the tenderness elicited over the joint, correlated with imaging findings, if any.

Fluoroscopic procedure: The patient was placed in the prone position with a pillow under the abdomen to correct the lumbar lordosis. The joint to be injected was located and marked. The x-ray tube was then slowly rotated till the joint appeared in profile as two parallel lines. After cleaning and draping, and administration of local anesthesia, a 22-G spinal needle was inserted in line with the x-ray beam till it contacted bone at the lip of the facet joint. With fine movements the needle tip could be made to enter the joint with a distinct 'give.' In the early cases, we confirmed the intra-articular position of the needle by injecting $0.5 \mathrm{ml}$ iohexol (Omnipaque ${ }^{\circledR}$; Amersham Health, New Jersey, USA) under fluoroscopy (figure 1).
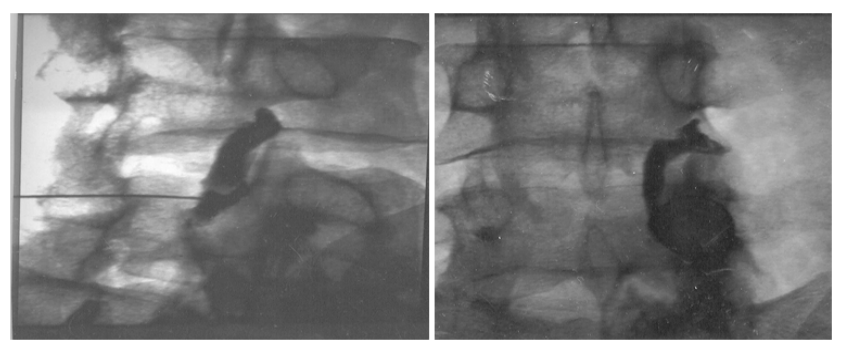

Fig (1). Fluoroscopy-guided lumbar facet joint injection at L4-5. The oblique spot image shows the intra-articular position of the needle.
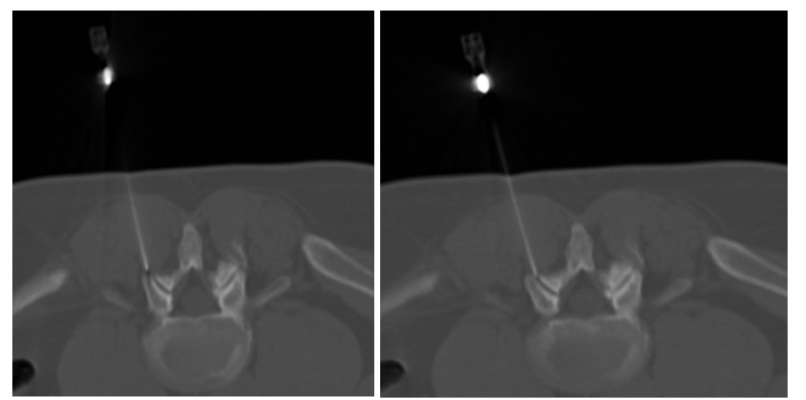

Fig (2). CT-guided facet injection. Axial CT scan in the prone position shows the needle positioned at the lip of the left L5-S1 facet joint.
Once the needle was in place, $0.5 \mathrm{ml}$ of $0.25 \%$ bupivacaine (a long-acting local anesthetic) and $0.5 \mathrm{ml}(20$ $\mathrm{mg}$ ) of methylprednisolone acetate were injected into the joint. The patient was observed for $1 \mathrm{~h}$ after the procedure to document pain relief and to monitor for allergic reactions.

CT-guided procedure: The patient was placed in the prone position and 5-mm axial sections were obtained at the level of interest to determine the entry site and the angle of approach (figure 2).

The entry site was marked on the skin and a $22-\mathrm{G}$ needle was advanced into the joint. The drug injection protocol was identical to the one used with fluoroscopic guidance.

The control group of 25 patients with chronic low back pain was subjected to medical treatment for one month in the form of central muscle relaxant (tizanidine - Sirdalud $\AA$ ) $2 \mathrm{mg}$ twice daily, antiiflammatory drugs (diclofenac -Flotac $\AA$ ) twice daily after meals or NSAID (Celebrex $\left.{ }^{\circledR}\right) 100 \mathrm{mg}$ twice daily in cases of gastritis, and steroids (betamethasone -

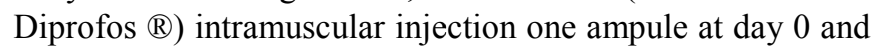
one ampule at day 15 .

Pain relief was assessed using a visual analog scale (VAS), with a score of 0 denoting 'no pain' and a score of 10 the 'worst pain possible' (figure 3).

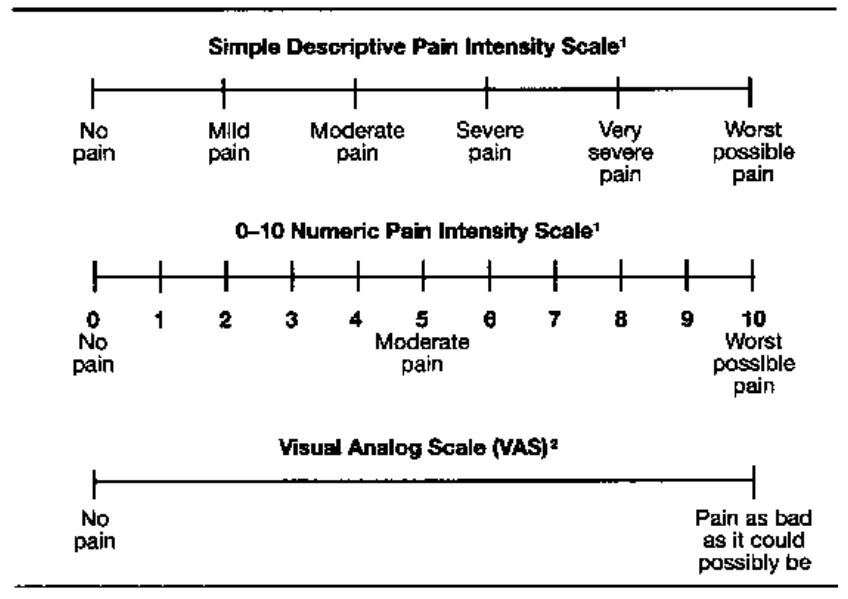

1)f used as a graphic rating scale a $10 \mathrm{~cm}$ baseline is recommendec

A $10-\mathrm{cm}$ beseline is recommended for VAS scales.

Fig (3). The visual analog scale for pain assessment (Source: Nature Clinical Practice Rheumatology 2007; 3: 610-618).

The VAS score was assessed before the procedure, 4 weeks after the procedure and, thereafter, at 12 , and 24 weeks. A reduction in the VAS score of $50 \%$ or more from the pretreatment score was considered as significant pain relief and the patient was labeled a 'responder.' These results were compared to a control group $(n=25)$ which were subjected to medical treatment for one month.

\section{Results}

A total of 146 facet joints were infiltrated in 50 patients, over a period of 2 years. The maximum number of infiltrations were at $\mathrm{L}_{4-5}(63 \%)$ followed by $\mathrm{L}_{3-4}(25 \%)$. Only a small proportion of the patients $(8 / 50)$ had an injection at a single level; most required injections were at multiple levels: 
18 infiltrations were at two levels, 16 at three levels, and 8 at four or more levels. The number of patients with significant postprocedure pain relief at 4,12 , and 24 weeks is detailed. Four weeks postprocedure, $88 \%$ reported significant pain relief. This number increased slightly reached a peak at 12 weeks, by which time as many as $92 \%$ patients had responded. However, the number of patients with pain relief declined to $64 \%$ at 24 weeks. The control group which was subjected to medical treatment showed pain relief in $40 \%$, $32 \%$, and $28 \%$ of patients at 4,12 , and 24 weeks respectively.

\section{Discussion}

The facet joints are known as the zygoapophyseal joints which are true synovial joints which are innervated by the medial branches of the dorsal rami. The presence of nociceptive nerve fibers in the synovium and fibrous capsule of the facet joints suggests that these joints may be a cause for LBP when they are stressed due to segmental instability, inflammatory synovitis, degenerative arthritis, or a combination of all of these ${ }^{(5,6)}$. Based on studies using controlled diagnostic blocks, it has now been conclusively proved that facet joints are a source of pain in as much as $15-45 \%$ of patients with $\operatorname{LBP}^{(2,5)}$.

Traditional approaches to diagnosis using physical examination, imaging and nerve contraction studies have been able to provide a specific diagnosis of facet joint as a source of pain in less than $30 \%$ of patients. ${ }^{(7)}$

No matter what the symptoms, one characteristic that all patients with facet syndrome have in common is relief of pain from injection of local anaesthetic. ${ }^{(8)}$

Unfortunately, there are no clinical or imaging findings to definitively diagnose facetal pain and very often the term 'facet syndrome' is used as a 'dustbin diagnosis' when nothing else fits. However, there are some features that are characteristic of facetal arthropathy. These include diffuse referred pain over the buttock and posterolateral thigh, exacerbation of pain with hyperextension or lateral bending, tenderness localized over one or more facet joints on deep pressure, and absence of root pain or neurological deficits. ${ }^{(4)}$

Imaging is not reliable for the diagnosis of facetal osteoarthritis since the changes seen on X-ray, CT, and MRI are equally common in patients with and without LBP, and most studies have failed to show a correlation between radiologic imaging findings and facet joint pain ${ }^{(9-11)}$

Typically, facet joint injections are performed as a part of a workup for back or neck pain. Since many patients do not have a readily identifiable cause for pain based on imaging studies and clinical evaluation, a stepwise process of different paraspinal injections is often performed. This process may include facet injections; epidural injections; selective nerve root blocks; and, in certain patients, discography. ${ }^{(12)}$

Facet joint injection with local anesthetic and steroid is the simplest and most common procedure for facet jointmediated pain. These infiltrations are diagnostic as well as therapeutic and the choice of guidance-whether CT or fluoroscopic - is largely a matter of personal preference and experience, as both are equally effective. ${ }^{(4,10)}$

Facet joint blocks have traditionally been performed by using fluoroscopic guidance, and this is still the most common method of localization. Since the advent of CT, and more recently CT fluoroscopy, these modalities are beginning to be more widely used to direct nerve root blocks. (12)

Proponents of fluoroscopy cite decreased procedure time and cost, whereas CT offers the advantages of no contrast material, more precise needle tip placement, and visualization of important vascular structures. Time limitations often prevent the use of traditional CT guidance, especially in busier units. The choice of technique should primarily be based on the physician's comfort, and ultimately, in skilled hands, there is probably no real difference. ${ }^{(12)}$

Two groups were included in this study, one control group of 25 patients that were subjected to medical treatment in the form of analgesics, muscle relaxant and steroids for one month. The other group of 50 patients was subjected to facet joint injection of steroid and analgesics under fluoroscopy or CT.

146 facet joints were included in the study represented by fifty patients. A mean of three facets were injected in every patient. This number of injected target facets per patient is similar to that was done in Bani et al. works. ${ }^{(13)}$ They injected 715 facets of 230 patients: 3.1 facet per patient. They collected this number of patients in five years from 1997 to 2001.

The age group ranged between 31-57 years old with mean age of 43 years. In comparison with previous studies, it is stated that the age incidence of facet joint syndrome is around 59 years. ${ }^{(9)}$ Bani et al. in 2002 published a study on the effect of facet joint block performed on 230 patients. The age ranged between 32 and 81 years with a mean age of 55 years. ${ }^{(13)}$

Male patients represented $48 \%$ of the sample, in disagreement with previous studies which showed increased incidence of this syndrome in males. ${ }^{(13)}$

L4-L5 level was the most frequently chosen level for injection representing $63 \%$ of the injected facet joints followed by L5-S1 level (25\%). L2-L3 and L3-L4 levels were the least injected levels, each representing $6 \%$ of the sample. The lower the injected level is, the more difficult to access the joint space under computed tomography due to the more coronal orientation of the facets caudally.

Indications of injection varied among the study sample: diffuse disc bulge with facet joint syndrome was the indication in $40 \%$, atypical pain of the facet joint syndrome $24 \%$, unilateral facet joint arthropathy $4 \%$, failed back surgery syndrome $12 \%$, and disc herniation with atypical sciatica $20 \%$.

This study did not include any patients with acute postoperative pain. The reason for this is that neurosurgery department does not include facet joint block as a part of the management protocol for these conditions. Also, acute postoperative radicular pain is not common: In the study sample 
of Bani et al. works in 2002 only $28 \%$ of it was due to this indication. Other indications in their study was: conservative management of facet joint hypertrophy associated with chronic low back pain and pseudoradicular manifestations ( $37 \%$ ), chronic post-operative pseudoradicular pain (16\%),

The immediate pain relief after the injection is attributed to the effect of the long-acting local anesthetic which interrupts the pain-spasm cycle. The corticosteroid begins to act by 1 week and by about 3 weeks the peak effect sets in. There may be a nonspecific synovitis present in many of these joints that is relieved by the anti-inflammatory action of corticosteroids. In many cases, rupture of the articular capsule during injection results in the drugs diffusing into the neural foramina too, thus, acting on the adjacent nerves as well. A simple physical effect, whereby inflammatory exudates or adhesions are cleared from the joint and the nerve root sleeve, may also play a role ${ }^{(14-16)}$.

Accurate assessment of pain is a prerequisite for the effective management of patients with LBP. Of the numerous tools available to assess pain, we adopted the numerical visual analog scale (VAS) since it is an objective measure and can be used to track serial changes. ${ }^{(16)}$ Many recent studies of facet joint injections have also used the VAS as the scoring system for pain, thus allowing us to compare our findings with other studies. Also in accordance with these studies, we considered a $50 \%$ reduction in the VAS score from the preprocedure level as indicating significant pain relief. $^{(15,17)}$

Literature describing the effectiveness of facet joint infiltrations is as abundant as it is controversial. Early studies of facetal infiltrations showed poor results and led to the conclusion that this is a nonspecific and ineffective method of treatment. ${ }^{(17,18)}$ It is possible that these disappointing results were due to improper patient selection, poor localization of the site for injection, or inadequacies in the volumes and types of drugs used. ${ }^{(4)}$ Other studies in recent years have reported encouraging results with facet joint infiltrations and good results of the pain relief. These results demonstrate significant short-term (1-12 weeks) pain relief in $62-74 \%$ of patients. Though the pain response gradually declines over time, even in the medium term (up to 24 weeks) as many as one-third of patients still experience significant pain relief. ${ }^{(11,15,19,20)}$

Our results showed short-term relief in $88-92 \%$ of patients and medium-term relief in $64 \%$, which compare favorably with the results of these earlier studies (table 1).

Table No.1. Assessment of pain relief after facet injections

\begin{tabular}{lll}
\hline Duration following injection & No. of patient 'responders' & Percentage \\
\hline 4 weeks & 44 / 50 & $88 \%$ \\
12 weeks & $46 / 50$ & $92 \%$ \\
24 weeks & 32 / 50 & $64 \%$ \\
\hline
\end{tabular}

In our opinion, the high number of responders in our study could be due to meticulous adherence to the patient selection criteria, with elicitation of paraspinal tenderness over the facet joint being the most important inclusion criterion. The presence or absence of facet joint arthropathy on imaging was not related to pain relief in any way, and the main factor associated with a successful outcome of facet joint interventions was clinically elicited paraspinal tenderness. ${ }^{(11,15,17)}$

The control group which was subjected to medical treatment showed short-term relief in $32 \%-40 \%$ of patients and medium-term relief in $28 \%$ with less pain relief in comparison to the experimental group that subjected to injection therapy (table 2).

Table No.2. Assessment of pain relief after medical treatment

\begin{tabular}{lll}
\hline Duration following injection & No. of patient 'responders' & Percentage \\
\hline 4 weeks & $10 / 25$ & $40 \%$ \\
12 weeks & $8 / 25$ & $32 \%$ \\
24 weeks & $7 / 25$ & $28 \%$ \\
\hline
\end{tabular}

Injections for spondylolysis are a modification of facetal injections, providing good response in most patients. Some workers feel that it is the fracture site which is painful and, accordingly, infiltrate the break in the pars interarticularis. ${ }^{(4)}$ Others have demonstrated that injection of the adjacent facet joint also involves the spondylolytic area and the technique can thus be used for therapeutic injections. ${ }^{(11,21)}$ The uniformly poor results with facet joint injections in patients with FBSS is because post-lumbar surgery pain is due to an interplay of numerous causes and facet joint injection addresses only one of them ${ }^{(22)}$ Due to the small number of FBSS patients in our series we could not derive any statistically significant information from our data.

The major complications of facet joint infiltrations are related to improper needle placement, bleeding, or infection. Complications include dural puncture, hematoma formation, spinal cord or neural trauma, spinal anesthesia, septic arthritis spondylitis, and chemical meningitis. ${ }^{(23)}$

Despite this long list and numerous anecdotal reports in literature, ${ }^{(24-26)}$ with meticulous attention to technique and sterility, these major complications can be eliminated.

\section{Conclusions}

CT-guided facet joint infiltration is a good method for treatment and diagnosis of lumbar facet joint syndrome. It can be repeated and has no severe side effects. The best results were seen in patients with spondylarthrosis of the facet joints. For patients with unspecific low back pain, facet joint injections are a very good diagnostic method, allowing definite exclusion of lumbar facet syndrome. Evidence-based guidelines, published in 2007, with respect to lumbar facet joint injections have convincingly demonstrated moderate evidence for short- and medium-term (up to 6 months) relief and limited evidence for long-term relief. ${ }^{(14)}$ Facetal injections are not curative; however, by abolishing pain for periods of up to 6 months they can decrease dependence on oral medications and facilitate early return to work. Since 
their clinical effect is for a limited duration and wanes after 6 months, they need to be repeated to maintain the pain relief. It is also important to adopt stringent criteria for diagnosing facet joint pain in order to avoid unnecessary and unwarranted injections. In conclusion, we have found that in carefully selected cases, lumbar facet block is a relatively simple, safe, and minimally invasive procedure that can be a valuable adjunct in the treatment of LBP.

\section{References}

[1] Cassidy JD, Côté P, Carroll LJ, Kristman V. Incidence and course of low back pain episodes in the general population. Spine. 2005;30:2817-23.Chang HJ, Burke AE, Glass RM. Gliomas. JAMA 2010; 303(10):1000.

[2] Manchikanti L, Singh V, Pampati V, Ghafoor AB, Fellows B, Damron KS, et al. Evaluation of the relative contributions of various structures in chronic low back pain. Pain Physician. 2001;4:308-16.

[3] Pang WW, Mok MS, Lin ML, Chang DP, Hwang MH. Application of spinal pain mapping in the diagnosis of low back pain: Analysis of 104 cases. Acta Anaesthesiol Sin. 1998;36:71-74.

[4] Silbergleit R, Mehta BA, Sanders WP, Talati SJ. Imagingguided injection techniques with fluoroscopy and CT for spinal pain management. Radiographics. 2001;21:927-39.

[5] Boswell MW, Colson JD, Sehgal N, Dunbar EE, Epter R. A systematic review of therapeutic facet joint interventions in chronic spinal pain. Pain Physician. 2007;10:229-53.

[6] Cavanaugh JM, Lu Y, Chen C, Kallakuri S. Pain generation in lumbar and cervical facet joints. J Bone Joint Surg Am. 2006;88:63-7.

[7] Galloway H. Image-guided spinal injection for diagnosis and therapy. Australasian Radiology 2003; 47, 219-225.

[8] Arti Chaturvedi, Sunil Chaturvedi, and Rajiv Sivasankar Image-guided lumbar facet joint infiltration in nonradicular low back pain Indian J Radiol Imaging. 2009 February; 19(1): 29-34.

[9] Schwarzer AC, Wang S, O'Driscoll D, Harrington T, Bogduk N, Laurent R. The ability of computed tomography to identify a painful zygapophysial joint in patients with chronic low back pain. Spine. 1995;20:907-12.

[10] Gilula LA, Lander P. Management of spinal pain with imaging-guided injection. Radiographics. 2003;23:189-91.

[11] Cohen SP, Hurley RW, Christo PJ, Winkley J, Mohiuddin MM, Stojanovic MP. Clinical predictors of success and failure for lumbar facet radiofrequency denervation. Clin J Pain. 2007;23:45-52.
[12] Wagner AL. Paraspinal injections: Facet Joint and Nerve Root Blocks. Available at: http://www.emedicine.com/radio/topic884.htm, Accessed October 7, 2005.

[13] Bani A, Spetzger U, and Gilsbach J. Indications for and benefits of lumbar facet joint block: analysis of 230 consecutive patients. Neurosurg. Focus 2002;13: 202-207.

[14] Boswell MV, Trescot AM, Datta S, Schultz DM, Hansen HC, Abdi S, et al. Interventional techniques: Evidence-based practice guidelines in the management of chronic spinal pain. Pain Physician. 2007;10:7-111.

[15] Gorbach C, Schmid MR, Elfering A, Hodler J, Boos N. Therapeutic efficacy of facet joint blocks. AJR Am J Roentgenol. 2006;186:1228-33.

[16] Mannion AF, Balagué F, Pellisé F, Cedraschi C. Pain measurement in patients with low back pain. Nat Clin Pract Rheumatol. 2007;3:610-8.

[17] Destouet JM, Gilula LA, Murphy WA, Monsees B. Lumbar facet joint injection: Indications, technique, clinical correlation, and preliminary results. Radiology. 1982;145:321-5.

[18] Lilius G, Harilainen A, Laasonen EM, Myllynen P. Chronic unilateral low-back pain: Predictors of outcome of facet joint injections. Spine. 1990;15:780-2.

[19] Shih C, Lin GY, Yueh KC, Lin JJ. Lumbar zygapophyseal joint injections in patients with chronic lower back pain. J Chin Med Assoc. 2005;68:59-64.

[20] Schulte TL, Pietila TA, Heidenreich J, Brock M, Stendel R. Injection therapy of lumbar facet syndrome: A prospective study. Acta Neurochir (Wien) 2006;148:1165-72.

[21] el-Khoury GY, Renfrew DL. Percutaneous procedures for the diagnosis and treatment of lower back pain: Diskography, facet joint injection, and epidural injection. AJR Am J Roentgenol. 1991;157:685-91.

[22] Wilkinson HA. In the failed back syndrome.etiology and therapy. 2nd ed. New York: Springer-Verlag; 1992. Introduction: Etiology, diagnosis, and therapy; pp. 1-3.

[23] Windsor RE, Pinzon EG, Gore HC. Complications of common selective spinal injec $\neg$ tions: Prevention and management. Am J Orthop. 2000;29:759-70.

[24] Nelemans PJ, deBie RA, deVet HC, Sturmans F. Injection therapy for subacute and chronic benign low back pain. Spine. 2001;26:501-15.

[25] Magee M, Kannangara S, Dennien B, Lonergan R, Emmett L, Van der Wall H. Paraspinal abscess complicating facet joint injection. Clin Nucl Med. 2000;25:71-3.

[26] Weingarten TN, Hooten WM, Huntoon MA. Septic facet joint arthritis after a corticosteroid facet injection. Pain Med. 2006;7:52-6. 